PO-0060 ANTI-THYROID ANTIBODIES AS A MARKER OF THYROID DISEASE IN PAEDIATRIC PATIENTS WITH DIABETES MELLITUS

M Ballesta, A Aramburu, C Juzga, G Carreras. Pediatrics, Hospital de La Santa Creu I Sant Pau, Barcelona, Spain

\subsection{6/archdischild-2014-307384.733}

Background and aims In a study carried out in adults with diabetes mellitus has been shown that monitoring thyroid function is cost effective in patients with positive anti-thyroid antibodies from the start of the illness; but the incidence of hypothyroidism is low in those patients who did not have antibodies from the begging.

Although autoimmune thyroiditis is rare in prepuberal age, the aim of our study was to determinate if the data obtained in adults correlates with the paediatric population diagnosed of diabetes mellitus and also to find out how many patients have thyroid antibodies during their follow up and when that positivization occurs.

Methods Retrospective descriptive study of 76 paediatric patients diagnosed of diabetes (between 1988 and 2013) at our hospital with available data of anti-thyroid antibodies (anti-thyroglobulin and anti-thyroperoxidase). Characteristics of age at the begging, Tunner stage, thyroglobulin antibodies, anti-thyroperoxidase, thyroid function, glycosylated haemoglobin and other antibodies were analysed.

Results 76 patients were included, 51\% were men and $48 \%$ women. The mean age of the study population at diagnosis was 8.15 years (1-16), Tunner stage 1 in $61 \%$ of cases, with mean baseline TSH of $2.9 \mathrm{mUI} / \mathrm{L}$. In an isolated case positive anti-thyroid antibodies were detected at the begging before puberty. Among all the patients with no anti-thyroid antibodies at the diagnosed of the illness, 3 patients had thyroid antibodies at prebuberal age and 6 after puberty, with a mean age 12.1 years at the time of the positivization (8-16 years).

Conclusions Most of the patients have negative anti-thyroid antibody titles at diagnosis, positivization often occurs after puberty or in adulthood, that suggests monitoring thyroid function is not cost effective in paediatric patients.

\section{PO-0061 ARE CLINICAL OR METABOLIC VARIABLES USEFUL TO DISTINGUISH BETWEEN AN INFANT OF A DIABETIC MOTHER AND SIMPLE MACROSOMIA OF THE NEWBORN?}

${ }^{1} \mathrm{R}$ Hentschel, ${ }^{1} \mathrm{M}$ Thill, ${ }^{1} \mathrm{~J}$ Trost, ${ }^{2} \mathrm{O}$ Schwab, ${ }^{3} \mathrm{M}$ Kunze, ${ }^{2} \mathrm{~A}$ Krause. ${ }^{1}$ Neonatology/Intensive Care Medicine, University Childrens Hospital Freiburg, Freiburg, Germany; ${ }^{2}$ Endocrinology/Diabetes, University Childrens Hospital Freiburg, Freiburg, Germany; ${ }^{3}$ Obstetrics/Gynecology, University Hospital Freiburg, Freiburg, Germany

\subsection{6/archdischild-2014-307384.734}

Background and aims Given the heritability of a diabetic predisposition and the need for a timely initiation of screening and preventive measures it seems prudent to identify infants from (a) a mother with preexisting diabetes mellitus (DM) or (b) with gestational diabetes (GDM), in contrast to infants with simple macrosomia (M).

Methods Identification of pregnant women with DM, GDM or fetal macrosomia in a level III obstetric department over a period of 5 months, using fetal ultrasound, medical history, oral glucose tolerance test and further variables. Collection of anthropometric and metabolic data from mother and infant.
Measurement of fructosamine, IGF, insulin, leptin and retinol binding-protein 4 from cord blood.

Results Mother-infant dyads with GDM ( $\mathrm{n}=64), \mathrm{M}(\mathrm{n}=18)$, DM (4) and control infants (C) $(n=47)$ were studied. Preconceptional weight, weight at delivery and BMI were significantly different between mothers with GDM and $\mathrm{M}$ infants versus $\mathrm{C}$ infants, respectively. Infants' anthropometric and blood glucose profile data were different from $C$ infants in GDM and $M$ group, but only anthropometric data were different between GDM and $\mathrm{M}$ infants. None of the 5 metabolic variables in cord blood was different between GDM and C group, but leptin and IGF were lower in GDM vs. $M$ and higher in $M$ vs. controls ( $p$ $<0.001)$.

Conclusions With the current screening tests cases of GDM may have been undetected in the $\mathrm{M}$ group. With adherence to screening guidelines and a high compliance of pregnant GDM women with medical recommendations metabolic differences between GDM and C infants might vanish and be restricted to anthropometric variables.

\section{PO-0062 TECHNOLOGICAL TOOLS TO OPTIMISE METABOLIC CONTROL IN ADOLESCENTS WITH TYPE 1 DIABETES}

${ }^{1}$ AB Ariza Jiménez, ${ }^{2}$ Leiva Gea, ${ }^{1} B$ Martín Tejedor, ${ }^{2}$ MJ Martinez-Aedo Ollero, ${ }^{2}$ JP López Siguero, ${ }^{1} \mathrm{~A}$ Urda Cardona. ${ }^{1}$ Pediatrics, Hospital Materno-Infantil. Hospital Regional Universitario de Málaga, Málaga, Spain; ${ }^{2}$ Pediatric Endocrinology, Hospital MaternoInfantil. Hospital Regional Universitario de Málaga, Málaga, Spain

\subsection{6/archdischild-2014-307384.735}

Introduction Flexible intensive therapy (FIT) emphasises in insulin administered before meals, adjusting the number of portions of carbohydrates and preprandialglycemic control. Accu - check expert (ACE) glucometer is a calculator with integrated data. It is necessary to introduce bolus insulin/carbohydrate ratio, factor sensitivity to insulin (FSI), ideal blood sugar and insulin duration adjusted by different time zones. It makes the calculation of insulin, managing in real-time the number of portions of carbohydrates and the glycemic control obtained. The patient must be trained how to change these parameters if it is required.

Aims and methods Prospective randomised intervention to determine the effectiveness of bolus calculator ACE in type 1 diabetic (D1) adolescents with FIT and values of HbA1c higher than 7\%. We measure glycosylated haemoglobin at 0,3 and 6 months.

Control group: 12 patients with D1 whose educational support involves counting portions and calculation of ratio and FSI before this intervention and at 3 months.

Case group: 12 patients with D1 who have received equal educational support as control group and specific training on bolus calculator, before baseline and at 3 months.

Results The groups are homogeneous in age, duration of diabetes and HbA1c before starting study. In both groups decreased HbA1c. There is a higher decrease in intervention group. The difference of $\mathrm{HbA} 1 \mathrm{c}$ is significative statistically in case group at 6 months.

Conclusions After the intervention both groups showed a decrease in HbA1c at 3 and 6 months, with higher difference in case group. This difference is clinically relevant and statistically significant in the case group when it is compared from baseline to 6 months.

\section{P0-0063 WITHDRAWN}

